\title{
Blind Doppler Compensation Scheme for Single Carrier Digital Underwater Communications
}

\author{
Goulven Eynard and Christophe Laot \\ Institut TELECOM; TELECOM Bretagne, CNRS LabSTICC, UMR 3192 \\ Technopole Brest-Iroise - CS 83818 - 29238 Brest Cedex 3, France \\ Email: Goulven.Eynard@ telecom-bretagne.eu, Christophe.Laot@telecom-bretagne.eu
}

\begin{abstract}
In this paper, we propose a dominant Doppler compensation technique for single carrier single input multiple output (SIMO) underwater acoustic communication. An initial pre-processing of the dominant Doppler is used to speed up the convergence rate of the adaptive receiver at the beginning of the transmission. Then, a tracking scheme is proposed to compensate for the residual dominant Doppler. The receiver scheme is tested in an experimental context. Successful communication is demonstrated at up to $4.926 \mathrm{kSymb} / \mathrm{s}$ with a transmitting platform moving up to $1.8 \mathrm{~m} / \mathrm{s}$. The system exhibits enhanced robustness for continuous-time communication with large Doppler shifts values and time-varying multipath channels.
\end{abstract}

\section{INTRODUCTION}

Phase coherent communications have proven in the past to be an efficient solution for high data rate underwater acoustic communications [1]. A relevant application is the transmission of images and data collected from an autonomous underwater vehicles (AUV's). Since a few years ago, GESMA (Groupes d'Etudes Sous-Marines de l'Atlantique at Brest, France), in collaboration with Telecom Bretagne, developped a robust acoustic link, called TRIDENT [2], [3].

Due to the low speed propagation environment $(c \approx$ $1500 \mathrm{~m} / \mathrm{s}$ ), the underwater acoustic channel exhibits high sensitivity to Doppler effect, affecting significantly synchronization (i.e timing and carrier recovery) algorithms [4]. Continuous-time communications between rapidly moving platforms assumes robust synchronization algorithms, able to track large and variable Doppler shifts. In this demanding context, specific reliable and highly adaptive synchronization algorithms are required [5]-[7].

It is well known that in an underwater acoustic channel, different paths can have different Doppler shifts [8]. However, the Doppler induced by the relative speed of the emitter has no common value compared to the differential Doppler spread between differents paths. Thus, as it is proposed in [9], [10], this paper is about compensating the dominant Doppler shift caused by relative motion that can be assumed to be the same on each hydrophones in a single input - multiple output (SIMO) context [11].

Classical Doppler estimation schemes derived from the maximum likelihood criteria can be obtained with a correlator bank [5]. However, this method requires high computationnal complexity. A more computationnally efficient method is presented in [6], that relies on the estimation of the compression/dilatation of the Doppler by measuring the length of a frame. The emitted frame are delimited with linear frequency modulated (LFM) signals which are known to be insensitive to Doppler effects.

This paper proposes a Doppler compensation scheme specifically adapted to compensate the dominant Doppler shift $\delta_{d}$ caused by the direct transmitter/receiver motion in an underwater acoustic channel. The estimation of the Doppler is extracted from a non data aided (NDA) feedforward symbol timing recovery scheme [12], slightly adapted to measure the temporal compression/dilatation of the received signal. Contrary to [6], this computationnally efficient method doesn't require any previous frame synchronization. This feedforward timing recovery scheme is known to be robust to ISI [13]. This robustness is critical when operating timing recovery before equalization. To the best knowledge of the authors, no adaptive timing recovery schemes are known to be insensitive to ISI. On the other side, no equalization schemes are insensitive to Doppler shift. This timing recovery algorithm appears thus to be the best candidate to compensate large Doppler shift. It must be stressed that if this initial Doppler compensation is not processed initially, classical adaptive equalization used with carrier phase recovery will not work properly.

When the symbol period and the carrier frequency are generated with the same quartz at the emitter and at the receiver, the carrier offset frequency (CFO) $f_{d}$ and the timing frequency offset (TFO) $\tau_{d}$ on the emitted signal are proportionnal. As the Doppler affects simulaneously carrier and timing frequency offset of the emitted signal, the estimation of the TFO can also be used to estimate the CFO. Doppler compensation can be operated jointly on carrier and timing using the same estimation. This pragmatic approximation exhibits very good results in terms of robustness when used before timing recovery and joint PLL/equalizer algorithms.

\section{IMPACT OF THE DOPPLER ON THE RECEIVED SIGNAL}

The relative Doppler shift $\delta_{d}$ is defined as the ratio of the source relative velocity $v$ to the propagation wave velocity $c$. Doppler effects can be accurately modeled as a time scaling (expansion/compression) of the signal waveform. $\delta_{d}$ will be assumed constant during the time of the estimation (approximatively $0.5 \mathrm{~s}$ ). This approximation is coarsely verified on sea trials in a shallow water context, when there is a relative movement between the emitter and the receiver. The received 
signal affected by a Doppler can be exprimed as:

$$
r(t)=s\left(\left(1-\delta_{d}\right) t\right)+w(t)
$$

where $s(t)$ is the emitted signal:

$$
s(t)=\Re\left\{\sum_{n=-\infty}^{+\infty} d_{n} g_{E}(t-n T) e^{j 2 \pi f_{c} t}\right\}
$$

and $w(t)$ is the noise. $f_{c}$ is the carrier frequency, $\left\{d_{n}\right\}$ are the emitted symbols and $g_{E}(t)$ is a pulse-shaping filter. $T$ is the symbol duration. The discrete-time signal obtained at the input of the timing recovery scheme can be written as:

$$
v\left(t_{m}\right)=\sum_{n=-\infty}^{+\infty} d_{n} g\left(\left(1-\delta_{d}\right) t_{m}-n T\right) e^{-j 2 \pi f_{c} \delta_{d} t_{m}}+b\left(t_{m}\right)
$$

where $b\left(t_{m}\right)$ is a complex baseband noise supposed to be gaussian and $g(t)$ is the convolution product of the transmitting filter $g_{E}(t)$ and the receiving filter $g_{R}(t)$ as well as the complex baseband channel impulse response $h(t)$ :

$$
g(t)=\left(g_{E} * h * g_{R}\right)(t)
$$

Assuming perfect timing recovery, the optimum sampling instant $t_{m}$ is exprimed as:

$$
t_{m}=m\left(T+\tau_{d}\right)+\epsilon[0]
$$

Where $\epsilon[0]$ is the initial delay and $\tau_{d}$ is the TFO:

$$
\tau_{d}=T \frac{\delta_{d}}{1-\delta_{d}}
$$

The following expression is obtained:

$v\left(t_{m}\right)=\left[d_{m} g(m T)+\sum_{n, n \neq m} d_{n} g((m-n) T)\right] e^{j 2 \pi f_{d} m T}+b\left(t_{m}\right)$

Where $f_{d}$ is defined as the CFO of the sampled signal that can be exprimed as:

$$
f_{d}=-f_{c} \frac{\delta_{d}}{1-\delta_{d}}
$$

Sampling at this optimal sampling instant doesn't compensate the CFO induced by the Doppler. Thus, in a underwater acoustic channel, a Doppler compensation scheme must account for simultaneously the TFO $\tau_{d}$ and the CFO $f_{d}$ in order to remove totally the Doppler effect. The estimation of the Doppler can be processed adversely on the CFO $f_{d}$ or on the TFO $\tau_{d}$ of the incoming signal, since there is a linear dependance between the two estimates. Classical algorithms uses an estimation of $f_{d}$ to extract an estimation of the Doppler. However, these algorithms may be sensible to ISI, and the estimation of the $\mathrm{CFO} f_{d}$ has a higher dynamic than the estimation of the TFO $\tau_{d}$. For these reasons, it is proposed in this paper to extract the timing frequency offset and use it to compensate the carrier frequency offset simultaneously as depicted on Fig.1.

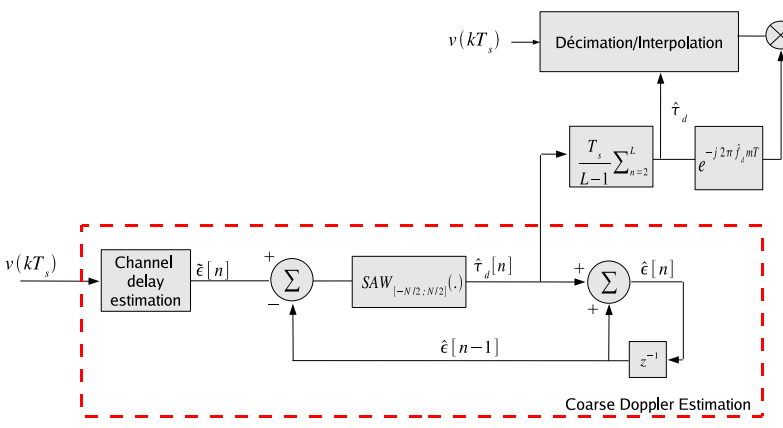

Fig. 1. Non data aided extraction and correction of the Doppler shift

\section{BLIND INITIAL DOPPLER COMPENSATION}

The Oerder and Meyr timing recovery algorithm provides a wrapped estimation of the channel delay $\epsilon[n]$ on a sub-block $n$, assuming the symbol period $T$ constant and known at the receiver [12]. The aim of the Doppler estimation is to provide an estimation of the new symbol period $T^{\prime}$ over $L$ sub-blocks of $K$ symbols duration where:

$$
T^{\prime}=T+\tau_{d}
$$

An estimation of $\tau_{d}$ can be obtained by computing the average of the difference between two consecutive estimations of the delay :

$$
\hat{\tau}_{d}=\frac{T_{s}}{L-1} \sum_{n=2}^{L} \hat{\tau}_{d}[n]
$$

where $\hat{\tau}_{d}$ is the estimation of $\tau_{d}$ and:

$$
\hat{\tau}_{d}[n]=\frac{1}{K}(\hat{\epsilon}[n]-\hat{\epsilon}[n-1])
$$

It must be stressed that the initial delay $\epsilon[0]$ is never compensated, since it doesn't rely on the Doppler effect but on the initial distance between the emitter and the receiver. This initial delay can be efficiently compensated using a fractionnaly spaced equalizer [1] or a classical timing recovery scheme [14]. Assuming the relative speed $v$ constant over the $L$ sub-blocks duration:

$$
\hat{t}_{m}=\hat{t}_{m-1}+T+\hat{\tau}_{d}
$$

Where $\hat{t}_{m}$ is the estimated sampling instant . Each sub-block is composed of $K$ symbols, using $N$ samples per symbol time. The Oerder et Meyr timing recovery algorithm does not provide an estimation of the delay $\hat{\epsilon}[n]$, but a wrapped estimation $\tilde{\epsilon}[n]$ where:

$$
\tilde{\epsilon}[n]=S A W_{[-N / 2 ; N / 2]}[\hat{\epsilon}[n]]
$$

and $S A W_{[-N / 2 ; N / 2]}$ is the sawtooth operation [12] that can be exprimed with the modulo operation:

$$
S A W_{[-N / 2 ; N / 2]}[\hat{\epsilon}[n]]=M O D_{N}(\hat{\epsilon}[n]-N / 2)-N / 2
$$

An unwrapping structure proposed in [12] can be used to solve this problem. The block diagram of the Doppler compensation scheme is plotted on Fig.1. 


\section{A. Blind initial SISO Doppler compensation}

As we employ a software timing recovery [14], [15], the received, filtered baseband signal $v(t)$ is first sampled at a constant rate $1 / T_{s}$, multiple of the symbol rate $1 / T$ before any operation of timing recovery.

$$
T=N T_{s}
$$

where $N$ is the oversampling factor. Usually, interpolation is used [7], [16], [17] to reconstruct the optimum sample aligned with the maximum eye opening from the sampled signal [18]. When the data rate is high, it is of interest to have an oversampling factor as low as possible in order to satisfy real-time specifications. However, when the data rate is low compared to those commonly encountered in radio communications, large values of $N$ can be taken. When this is the case $(N \geq 16)$, the maximum distance between the optimum sampling instant and the nearest effectively sampled instant can be considered negligible [14]. The gain of precision obtained from interpolation is weak compared to the additional cost in computational complexity. In this case, a simple adaptive decimation of the signal can be operated from the parameter value $\hat{\tau}_{d}$. From Eq. (12):

$$
\hat{t}_{m} \approx\left\lfloor t_{m} / T_{s}\right\rfloor T_{s}=\left\lfloor\left(t_{m-1}+T+\hat{\tau}_{d}\right) / T_{s}\right\rfloor T_{s}
$$

where $\lfloor$.$\rfloor is the function returning the floor of a number. As it$ has been proposed before, the linear relation betwen the TFO and the CFO can be exploited to obtain a coarse estimation of $f_{d}$ :

$$
\hat{f}_{d}=-\hat{\tau}_{d} f_{c} / T
$$

Where $\hat{f}_{d}$ represents the estimation of the CFO $f_{d}$. The Doppler estimation and compensation scheme for the SISO case is plotted on Fig.1. An extension of this algorithm to the SIMO case is straighforward and is presented on section IV.

\section{B. Blind initial SIMO Doppler compensation}

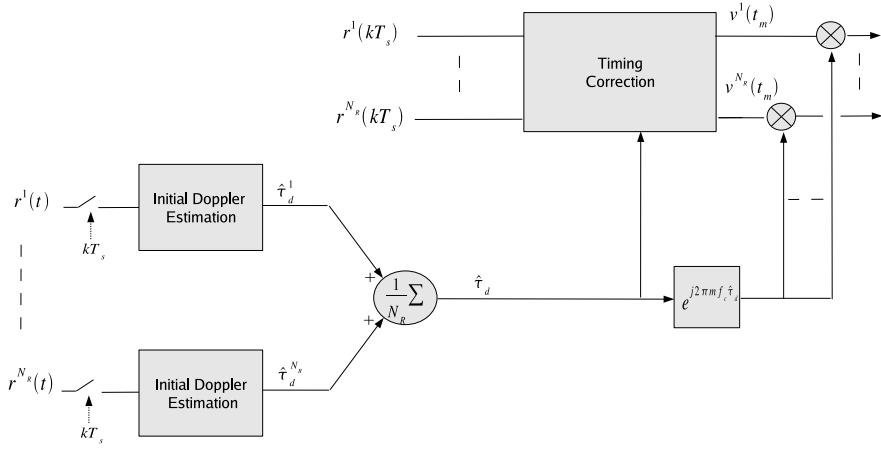

Fig. 2. SIMO Doppler compensation

As the compensation is here on the dominant Doppler shift, the Doppler shift can be assumed the same on each antenna. In order to have the spatial diversity gain, the estimation obtained on each antenna are averaged (Fig.2). Let $\hat{\tau}_{d}^{i}$ be the estimation of the TFO on the antenna $i$. The estimation of the dominant TFO can be obtained:

$$
\hat{\tau}_{d}=\frac{1}{N_{R}} \sum_{i=1}^{N_{R}} \hat{\tau}_{d}^{i}
$$

An interesting point is that each estimate $\hat{\tau}_{d}^{i}$ can be added coherently even in the case of a timing phase difference between each antenna. This is different of a correlation-based Doppler estimation that requires to first localize the maximum of the correlation peak on each antenna and then coherently sum each maximum obtained.

\section{SIMO DOPPLER COMPENSATION TRACKING SCHEME}

Once the initial Doppler compensation scheme is processed, an adaptive Doppler compensation scheme is needed at the receiver in order to track the time-varying dominant Doppler. To remove the slowly time-varying ISI due to channel multipath, an adaptive SIMO soft DFE equalizer, jointly optimized with a phase locked loop (PLL), presented in [19] is used. As suggested in [7], it can be proven that an adaptive estimation of the residual dominant CFO $f_{r}[\mathrm{~m}]$ can be extracted from the PLL. Supposing the estimation $\hat{f}_{r}[\mathrm{~m}]$ of the residual dominant CFO available at the output of the PLL, the correction of the instantaneous dominant CFO on each antenna $\hat{f}_{D}[\mathrm{~m}]$ can be exprimed as:

$$
\hat{f}_{D}[m]=\hat{f}_{d}+\hat{f}_{r}[m]
$$

Moreover, an estimation of the residual TFO $\tau_{r}[m]$ can be obtained using Equ. 6 and Equ. 8:

$$
\hat{\tau}_{r}=-\hat{f}_{r}[m] T / f_{c}
$$

Thus, an adaptive estimation of the dominant TFO can also be obtained:

$$
\hat{\tau}_{D}[m]=\hat{\tau}_{d}+\hat{\tau}_{r}[m]
$$

It is important to note that it is principaly the dominant TFO present on each antenna that is responsible of the failure of classical adaptive equalizations schemes used in radiocommunication. The residual timing phase, that can be different between each antenna, can be removed using fractionnally spaced equalizer [1] or a classical timing recovery scheme [14]. The SIMO Doppler compensation tracking scheme jointly jointly optimized with the initial Doppler compensation scheme is depicted on Fig.3.

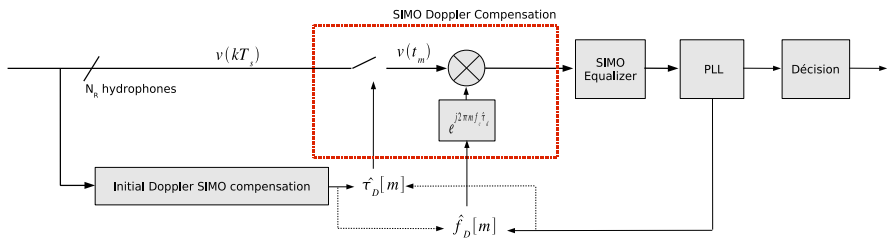

Fig. 3. SIMO Doppler compensation tracking scheme 


\section{Simulation Results}

The evaluation of the dominant Doppler shift compensation scheme is performed from the database collected by the GESMA in collaboration with TELECOM Bretagne during series of trials in the Atlantic ocean. The receiver employed a $N_{R}=4$ omnidirectional hydrophones. Carrier frequency of $f_{c}=35 k H z$ was used. The modulation technique was QPSK. The transmission range is 1500 meters. The AUV is $10 \mathrm{~m}$ below the surface in about $20 \mathrm{~m}$ deep water. The symbol rate used is $4.926 \mathrm{kSymb} / \mathrm{s}$. The relative speed observed between the boat and the AUV was estimated to $v=-1.8 \mathrm{~m} / \mathrm{s}$. Initial Doppler estimation is processed on $L=50$ sub-blocks of $K=100$ symbols and $N=28$ samples per symbol. As the emitted data are not known, the estimated mean square error (MSE) at the output of the equalizer is used as a measure of performance of the transmission rather than the bit error rate (BER). It can be shown than a residual estimated MSE of $-10 d B$ at the output of an equalizer can be approximated as having a AWGN channel with an SNR of $10 d B$. The estimated MSE is exprimed as:

$$
E M S E[k]=\beta E M S E[k-1]+(1-\beta)|\hat{d}[k]-y[k]|^{2}
$$

where $\beta$ is the forgetting factor, choosen here equal to 0.99 . The evolution of the estimated TFO $\hat{\tau}_{d}[m]$ is also plotted. As a matter of comparison, the proposed receiver is compared to the receiver proposed in [3]. Results are plotted on Fig.4 and Fig.5. It can be observed that when no Doppler compensation scheme are used, the adaptive equalizer has difficulty to converge, whereas on Fig.5 the coarse initial Doppler estimation $\hat{\tau}_{d}$ ensure and speed up the convergence of the TFO $\hat{\tau}_{D}[m]$. The good estimation of the TFO ensures the good convergence of the overall system as the estimated mean square error at the ouptut of the equalizer indicates. A good behavior of the overall receiver using the Doppler compensation scheme was observed during the time of the transmission (several minutes).

\section{CONCLusions}

In this paper, we have shown that reliable underwater acoustic communication over a long time duration and a difficult environment is possible using a classical PLL/equalizer structure and a specific Doppler compensation scheme. A computationnaly efficent coarse Doppler pre-compensation scheme is presented in this paper. Compared to other classical algorithms where correlation is needed, no previous frame synchronization are required. Moreover, each estimation processed on each antenna can be added coherently even in the case of different time delays on the antenna. Further study needs to be done however to remove the nonuniform Doppler shift present on each path of the channel.

\section{REFERENCES}

[1] M. Stovanovic, J. A. Catipovic, and J. G. Proakis, "Phase coherent digital communications for underwater acoustic channels," IEEE J. Oceanic Eng., vol. OE-16, pp. 100-110, Jan. 1994.
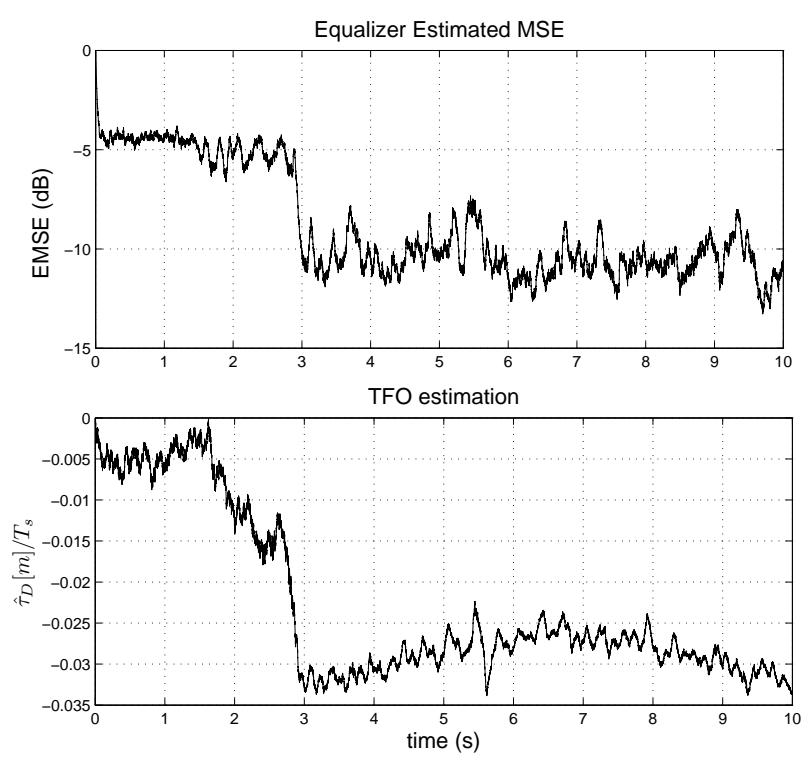

Fig. 4. Transmission performance without Doppler compensation
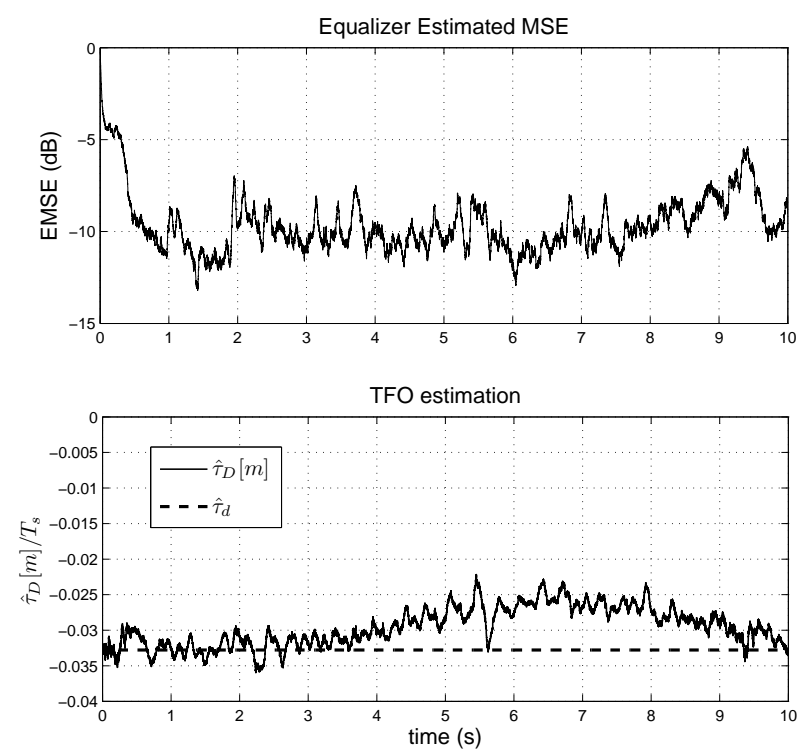

Fig. 5. Transmission performance with the proposed blind Doppler compensation scheme

[2] G. Lapierre, N. Beuzelin, J. Labat, J. Trubuil, A. Goalic, S. Saoudi, G. Ayela, P. Coince, and S. Coatelan, "1995-2005 : ten years of active research on underwater acoustic communications in brest," in in Proc. of OCEANS'05, Brest, France, 2005, pp. 425- 430.

[3] J. Trubuil, G. Lapierre, J. Labat, N. Beuzeulin, A. Goalic, and C. Laot, "Improved auv autonomy provided by an underwater acoustic link," in Proc. ISOPE'06, San Francisco, USA.

[4] J. Preisig, "Acoustic propagation considerations for underwater acoustic communications network development," in in Proc. of WUWNet'06, New-York, USA, 2006, pp. 1-5.

[5] M. Johnson, L. Freitag, and M. Stovanovic, "Improved doppler tracking and correction for underwater acoustic communications," in Proc. ICASSP'97, Munich, Germany, pp. 575-578.

[6] B. Sharif, J. Neasham, O. Hinton, and A. E. Adams, "A computationaly efficient doppler compensation system for underwater acoustic commu- 
nications," IEEE J. Oceanic Eng., vol. OE-25, pp. 52-61, Jan. 2000.

[7] M. Stovanovic and L. Freitag, "Integrated doppler tracking and efficient resampling for phase coherent acoustic communication. [online] available: www.mit.edu/militsa/resources/pdfs/joedop.pdf."

[8] J. Dhanoa and R. Ormonroyd, "Combined differential doppler and time delay compensation for an underwater acoustic communication system," in Proc. of OCEANS'02, 2002, pp. 581- 587.

[9] T. Oberg, B. Nilson, O. Olofson, M. L. Nordenvaad, and E. Sangfelt, "Underwater communication link with iterative equalization," in Proc. of OCEANS'06, 2006, pp. 1-6.

[10] B. Li, S. Zhou, M. Stovanovic, L. Freitag, and P. Willet, "Multicarrier communication over underwater acoustic channels with nonuniform doppler shifts," IEEE J. Oceanic Eng., 2008 (to appear).

[11] J. Tao, Y. Zheng, C. Xiao, T. Yang, and W.-B. Yang, "Channel estimation, equalization and phase correction for single carrier underwater acoustic communications," in Proc. of OCEANS'08, Kobe, Japan, 2008.

[12] M. Oerder and H. Meyr, "Digital filter and square timing recovery," IEEE Trans. Commun., vol. COM-36, pp. 605-612, May 1988.

[13] G. Eynard and C. Laot, "Non data aided timing recovery algorithm for digital underwater communications," in Proc. of OCEANS'07, Aberdeen, Scotland, 2007.

[14] H. Meyr, M. Moeneclaey, and S. Fechtel, Digital Communication Receivers: Synchronization, Channel Estimation, and Signal Processing. New York: Wiley, 1998.

[15] F. Gardner, Phaselock Techniques. 2nd edition, Wiley, 1966.

[16] — "Interpolation in digital modem part i: fundamentals," IEEE Trans. Commun, vol. 41, pp. 501-507, March 1993.

[17] L. Erup, F. Gardner, and R. Harris, "Interpolation in digital modem part ii: implementation and performance," IEEE Trans. Commun., vol. 41, pp. 998-1008, March. 1993.

[18] U. Mengali and A. D’Andrea, Synchronization Techniques for Digital Receivers. Springer, 1997.

[19] J. Labat and C. Laot, "Blind adaptive multiple-input decision feedback equalizer with a self-optimized configuration," IEEE Trans. Commun., vol. 49, pp. 646-654, April 2001. 